\title{
An Approach toward the Efficient Indexing and Retrieval on Medical X-Ray Images
}

\author{
Sumathi Ganesan \\ Department of Computer Science and \\ Engineering Annamalai University \\ Annamalai Nagar - 608 002, India.
}

\author{
T.S. Subashini \\ Department of Computer Science and \\ Engineering Annamalai University \\ Annamalai Nagar - 608 002, India.
}

\begin{abstract}
Today content-based image retrieval (CBIR) has become one of the most active areas of research in computer vision. With rapid advances in digital imaging modalities, the use of CBIR to search for the clinically relevant and visually similar medical images is highly felt nowadays. This paper proposes a system for content based image retrieval of X-ray images. The six classes of X-ray images used for this work are from the IRMA ImageCLEFmed 2008 database. Discrete Cosine Transform (DCT) coefficients were used as features and the $\mathrm{X}$-rays were classified using Support Vector Machine (SVM). The classified images along with the features were stored in the database using hierarchical index structure. Euclidean distance is used as the metric for retrieving the top three images from the database relevant to the given query image
\end{abstract}

\section{INTRODUCTION}

Radiologists often in their clinical practice of reading and interpreting medical images refer to and compare similar cases with verified diagnostic results in their decision making. However, searching for and identifying thesimilar reference cases (or images) from the large and diverse clinical databases is a quite difficulttask. The advance in digital technologies for computing, networking, and database storage has enabledthe automated searching for clinically relevant and visually similar medical examinations (cases) to thequeried case from the large image databases [1].

Current work in image processing, medical informatics and information retrieval domains provides building blocks that can markedly increase the relevance of content-based image retrieval (CBIR) to radiology practice in the future [2].

In this work an effort has been made to automatically search and retrieve X-rays similar to the given query image. A method is proposed to automatically classify X-rays at the macro level (coarse level) using DCT and SVM. The X-ray images are classified into major classes namely chest, head, foot, palm, spine and neck using the extracted features. These features called visual signature along with the corresponding $\mathrm{X}$-ray is automatically indexed and stored in the database using hierarchical indexing structure. These visual signatures areused to rank the images based on Euclidean distance similarity measures for retrieving similar images.

The X-ray images used for this work are from the IRMA ImageCLEFmed 2008 database. This collection compiles anonymous radiographs, which have been arbitrarily selected from routine at the Department of Diagnostic Radiology, Aachen University of Technology (RWTH), Aachen, Germany. The imagery represents different ages, genders, view positions and pathologies. Therefore, image quality varies significantly. All images were downscaled to fit into a $512 \times 512$ bounding box maintaining the original aspect ratio [13].

This paper is organized as follows. Section 2 gives an overview of the literature. The proposed methodology is given in section 3. Experimental results are dealt with in section 4. Section 5 concludes the paper.

\section{PREVIOUS WORK}

Image classification is a pattern recognition problem that involves three steps, feature extraction, feature selection by dimension reduction and classification (the training of a classifier with extracted features)[11].The work in [9] has integrated the use of a probabilistic multiclass support vector machine and fuzzy c-mean clustering for categorization and pre-filtering of images. An image representation and matching framework for image categorization in medical image archives is presented in [10] where a continuous and probabilistic image representation scheme using Gaussian mixture model (GMM) along with information-theoretic image matching via the Kullback-Leibler measure is proposed. For feature selection Principal component analysis (PCA), linear discriminant analysis (LDA), and independent component analysis (ICA) are used in [5]. In [14] SVM are used for Classification. The work in [17] has extracted shape features such as Fourier descriptor, invariant moments and Zernike moments and Bayesian rule classifies 28 classes of Xray images. In [18] shows that LBP and BoW provides better performance of $90 \%$ accuracy. Fuzzy classifier gives $78.61 \%$ class wise accuracy and $80.38 \%$ global accuracy [19].

\section{PROPOSED METHOD}

The block diagram of the proposed method is shown in Fig.1.

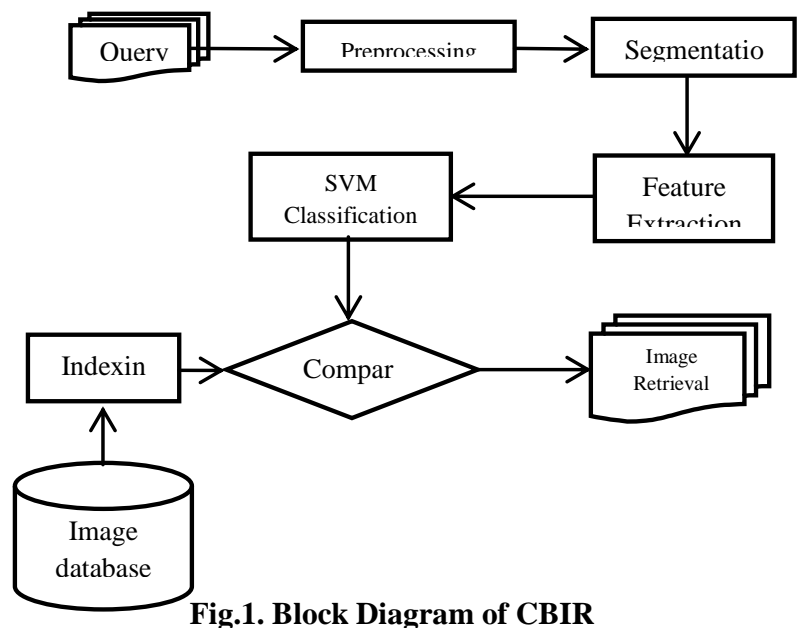




\subsection{PREPROCESSING}

Median filtering and histogram equalization [3] is applied to the cropped X-ray as a preprocessing step to reducing noise and to enhance the image. Then the images are resized and uniformly oriented to avoid unnecessary computations and misclassifications.

\subsection{SEGMENTATION}

Segmentation is accomplished through adaptive thresholding and connected component labeling. By CCL, various connected regions on the binary image are obtained and only the biggest connected region which is the body part examined is retained and other regions are eliminated. [4] Identification of image features that are relevant for medical diagnosis is not part of this work, since this work aims at a coarse level classification for determining the examined body part. Comparing CCL image with the original filtered image, the pixel value of the region of interest is restored from the original image.

\subsection{FEATURE EXTRACTION AND DIMENSION REDUCTION}

The segmented image is transformed into a reduced representation set of features using DCT and PCA.

\subsubsection{THE DISCRETE COSINE TRANSFORM}

Like other transforms, the Discrete Cosine Transform (DCT) attempts to de-correlate the image data. After de-correlation each transform coefficient can be encoded independently without losing compression efficiency.

The most common DCT definition of a 1-D sequence of length $\mathrm{N}$ is

$C(u)=\alpha(u) \sum_{x=0}^{N-1} f(x) \cos \left[\frac{\pi(2 x+1) u}{2 N}\right]$

for $\mathrm{u}=0,1,2, \ldots, \mathrm{N}-1$. Similarly, the inverse transformation is defined as

$$
f(x)=\sum_{x=0}^{N-1} \alpha(u) C(u) \cos \left[\frac{\pi(2 x+1) u}{2 N}\right]
$$

for $\mathrm{u}=0,1,2, \ldots, \mathrm{N}-1$.

In both equations (1) and (2) $\alpha(\mathrm{u})$ is defined as

$\alpha(u)= \begin{cases}\sqrt{\frac{1}{N}} & \text { for } u=0 \\ \sqrt{\frac{2}{N}} & \text { for } u \neq 0\end{cases}$

It is clear from (1) that

$$
C(u=0)=\sqrt{\frac{1}{N}} \sum_{x=0}^{N-1} f(x) .
$$

$\mathrm{u}=0$

Thus, the first transform coefficient is the average value of the sample sequence. In literature, this value is referred as the DC coefficients. All other transform coefficients are called the AC coefficients.

The 2-D DCT is a direct extension of the 1-D case and is given by
$C(u, v)=\alpha(u) \alpha(v) \sum_{x=0}^{N-1} \sum_{x=0}^{N-1} f(x, y) \cos \left[\frac{\pi(2 x+1) u}{2 N}\right] \cos \left[\frac{\pi(2 y+1) v}{2 N}\right]$

for $\mathrm{u}, \mathrm{v}=0,1,2, . ., \mathrm{N}-1$ and $\alpha(\mathrm{u})$ and $\alpha(\mathrm{v})$ are defined in (3).

Discrete Cosine transforms is applied to the segmented region to extract coefficients which are used as features for classification. In an image, most of the energy will be concentrated in the lower frequencies, so if the image is transformed into its frequency components and the higher frequency coefficients are discarded, the amount of data needed to describe the image can be reduced. DCT's energy compaction efficiency is so great that can reduce the amount of data needed to describe the image without sacrificing too much image quality.

\subsubsection{FEATURE REDUCTION USING PCA}

Subsequently PCA is applied to obtain the most relevant 30 coefficients which form the feature vector or signature of the image. Principal component analysis (PCA) involves a mathematical procedure that transforms a number of (possibly) correlated variables into a (smaller) number of uncorrelated variables called principal components.[5] The first principal component accounts for as much of the variability in the data as possible, and each succeeding component accounts for as much of the remaining variability as possible. In this work PCA is applied to obtain the most relevant 30 DCT coefficients from a large number of coefficients which are irrelevant and redundant.

\subsection{SVM CLASSIFICATION}

Classification is typically applied for either automatic annotation, or for organizing unseen images into broad categories for the purpose of retrieval. It is advantageous to go for image classification when the image database is well specified, and labeled training samples are available. Domainspecific database such as medical image database is an example where classification can be beneficial.

SVMs are powerful machine learning techniques for classification and regression. They were developed by Vapnik [6], and are based on statistical learning theory. This gave rise to a new class of theoretically elegant learning machines that use a central concept of support vectors and kernels for a number of learning tasks. Kernel machines provide a modular framework that can be adapted to different tasks and domains by the choice of the kernel function and the base algorithm. In the parlance of SVM literature, a predictor variable is called an attribute, and a transformed attribute that is used to define the hyperplane is called a feature. The vectors near the hyperplane are the support vectors. [7,12]. Fig. 2 presents an overview of the SVM process.

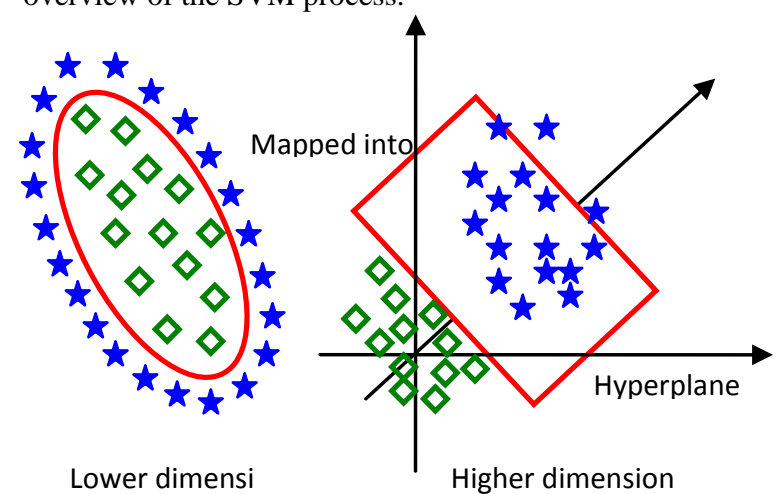

Fig. 2 Overview of SVM 


\subsection{INDEXING}

Once image features were extracted and classified, they should be indexed and matched against each other for retrieval. In this paper, hierarchical indexing technique[16] is used and the class label is used to index into the database. A total of 300 images belonging to six class of X-rays and each class consisting of 50 imageswere used to test the efficacy of the proposed CBIR system.

\subsection{RETRIEVAL}

A distance measure is a function that computes and returns a value corresponding to the similarity between two objects according to some pre-defined criteria. In this work, Euclideandistance method [15] is used to retrieve similar images from the created indexed database. When query image is given, the DCT feature ofthat image is identified and classified with supportvector machine into any of the six class of X-ray. This class label obtained is used to index into the databaseof the corresponding class. Then searching and retrieval is carried out by means of Euclidean distance method [15]. Visual signatures are found out and the corresponding similar images are displayed on the user interface screen. Using Euclidean distance the query images' visual signature is compared with the visual signatures of other images stored in the database. The shortest distance will beconsidered as best matching image in that matching process. Thedistance $\mathrm{d}$ between two image feature vectors is calculatedusing the following equation.In the data

$$
d=\sqrt{\sum_{i=0}^{N} f_{q}\left((i)-f_{d}(i)\right)^{2}}
$$

where, $f_{q}(i)$ is the $i^{\text {th }}$ feature vector of the query image and $f_{d}(i)$ is the $i^{\text {th }}$ feature vector of the database image.

\section{EXPERIMENTAL RESULTS}

Initially the X-ray images are preprocessed and segmented using connected component labeling. Then the most relevant 30 DCT coefficients are taken as visual signature and classified using SVM which gave an accuracy of $95.82 \%$.

A database consisting of the X-ray image along with its visual signature is created using hierarchical indexing structure. Euclidean distance is used as a similarity measure to search and retrieve the top three images similar to the given query image. 30 test images, five belonging to each of the six classes of X-ray images are used to test this proposed system. Table 1 shows the results of the proposed work and using this; the precision is calculated as $86.6 \%$. Precision refers to the percentage of retrieved pictures that are relevant to the query [8]. Table 2 shows the comparison between proposed work and previous works.

The screenshots of the experimental results are shown in Figs. $3,4.1 \& 4.2$.
Table 1: Results of proposed work

\begin{tabular}{|l|c|c|c|c|c|c|}
\hline & Chest & Foot & Head & Palm & Neck & Spine \\
\hline $\begin{array}{l}\text { No. of } \\
\text { test } \\
\text { images }\end{array}$ & 50 & 50 & 50 & 50 & 50 & 50 \\
\hline $\begin{array}{l}\text { No. of } \\
\text { correctly } \\
\text { retrieved } \\
\text { Images }\end{array}$ & 50 & 50 & 40 & 40 & 50 & 30 \\
\hline
\end{tabular}

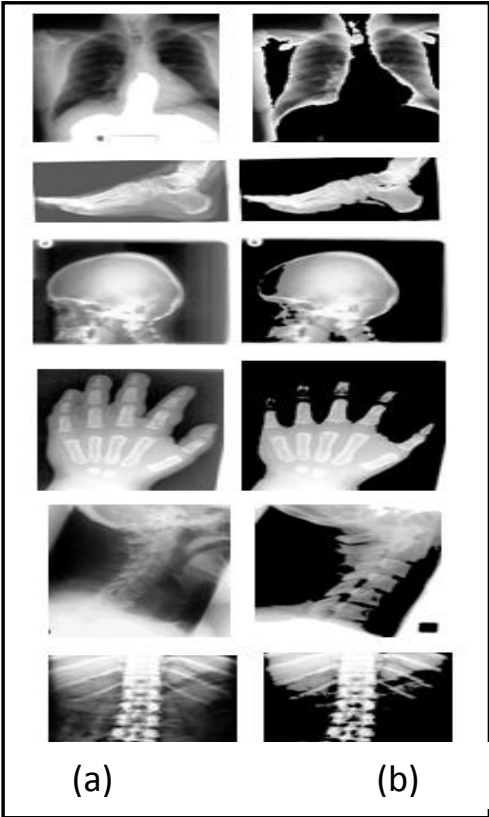

Fig 3: (a) preprocessed image (b) Segmented image

Table 2 : Comparison between proposed work and previous works

\begin{tabular}{|l|l|l|c|l|}
\hline S.No. & $\begin{array}{l}\text { Features } \\
\text { extracted }\end{array}$ & Classifier & Accuracy & Reference \\
\hline 1. & $\begin{array}{l}\text { Fourier } \\
\text { descriptor } \\
\text { Zernike } \\
\text { moments }\end{array}$ & $\begin{array}{l}\text { Bayesian } \\
\text { rule }\end{array}$ & $82.87 \%$ & {$[17]$} \\
\hline 2. & LBP, BoW & SVM & $90 \%$ & {$[18]$} \\
\hline 3. & Fuzzy & $\begin{array}{l}\text { Texture } \\
\text { and Shape }\end{array}$ & $80.38 \%$ & {$[19]$} \\
\hline 4. & $\begin{array}{l}\text { GLCM and } \\
\text { Zernike } \\
\text { Moment }\end{array}$ & SVM & $95.82 \%$ & $\begin{array}{l}\text { Proposed } \\
\text { work }\end{array}$ \\
\hline
\end{tabular}




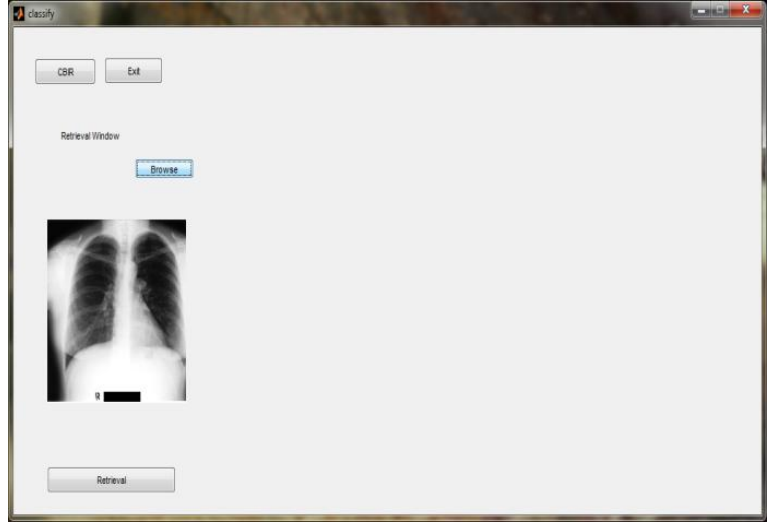

Fig 4.1 Loaded Query Image.

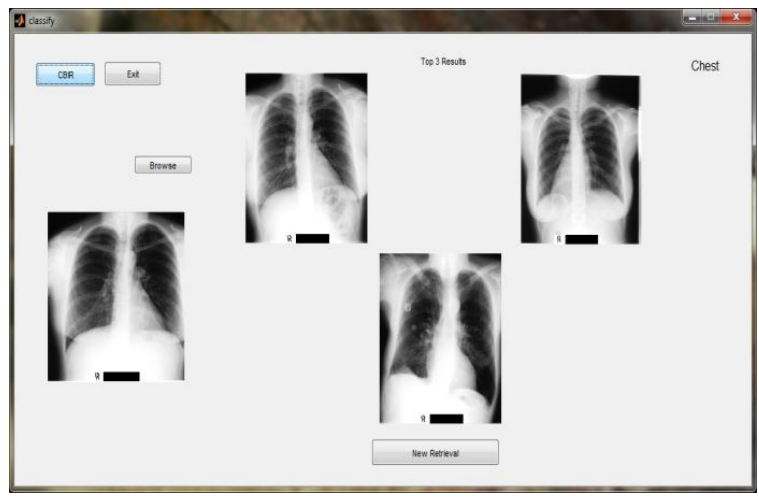

Fig 4.2 Top 3 retrieved Images

\section{CONCLUSION}

In this work an attempt has been made to retrieve similar Xrays based on the content of the query image. The most relevant Discrete Cosine Transform (DCT) coefficients were used as features and the $\mathrm{X}$-rays were classified using Support Vector Machine (SVM) which gave a classification accuracy of $95.82 \%$. Based on classification of X-ray images an indexed database is created using hierarchical indexing structure.Euclidean distance is used as the metric for retrieving the top three images from the database relevant to the given query image. The precision rate of $86.6 \%$ shows that this system can be effective in content based image retrieval of X-ray images.

\section{REFERENCES}

[1] Liu Y, Zhang D, Lu G, Ma WY. A survey of contentbased image retrieval with high-level semantics. Pattern Recognition 2007; 40:262-82.

[2] Lehmann TM, Guld MO, Deselaers T, Keysers D, Schubert H, Spitzera K, et al. Automatic categorization of medical images for content-based retrieval and data mining. Comput Med Image Graph 29:143-55, 2005.

[3] Rafael C.Gonzalez\& Richard E.Woods, "Digital Image Processing", Second Edition, 2005.

[4] Rafael C. Gonzalez, Richard E. Woods \& Steven L. Eddins, "Digital Image Processing Using MATLAB", Pearson Education 2007.
[5] Principal

component

analysis:

www.fon.hum.uva.nl/praat/manual/principal_component _analysis.html

[6] V.Vapnik, Statistical Learning Theory, Wiley, New York, 1998.

[7] SVM - Support Vector Machines: www.dtreg.com.svm.html

[8] Marina Sokolova, Nathalie Japakowicz and Stan Szpakowicz, "Beyond Accuracy, F-score and ROC: a family of Discriminant measures for performance evaluation",www.etud.iro.umontreal.ca/nsokolovm/austr alia06.pdf/ 2006.

[9] Rahman MM, Bhattacharya P, Desai BC. A framework for medical image retrieval using machine learning and statistical similarity matching techniques with relevance feedback. IEEE Trans Inf Technol Biomed 2007;11(1):58-69.

[10] Greenspan H, Pinhas AT. Medical image categorization and retrieval for PACS using the GMM-KL framework. IEEE Trans InfTechnol Biomed 2007;11(2):190-202.

[11] Jain AK, Duin PW, Mao J. Statistical pattern recognition - a review. IEEE Trans Pattern Anal Mach Intell 2000; 22(1):4-36.

[12] K.Vaidehi T. S.Subashini V. Ramalingam S. Palanivel M. Kalaimani, "Transform based approaches for Palmprint Identification", International Journal of Computer Applications (0975 - 8887) Volume 41- No.1, March 2012.

[13] Lehmann, T "IRMA X- Ray Library url: http://irmaproject.org.

[14] D.C. Tao, X.O. Tang, X.L. Li, X.D. Wu, Asymmetric bagging and random space for support vectormachinesbasedrelevance feedback in image retrieval, IEEE Trans. Pattern Analysis and Machine Intelligence, vol.28, n07, July 2006.

[15] Content based Image Retrieval for Medical Images using Canny Edge Detection Algorithm,International Journal of Computer Applications (0975 - 8887)Volume 17No.6, March 2011.

[16] Datta,R.,Joshi,D.,Li,J.,andWang,J.Z.Image retrieval: Ideas, influences, and trends of the new age. ACM Comput. Surv. 40, 2, Article 5 (April 2008).

[17] Fesharaki, Nooshin Jafari, and Hossein Pourghassem. "Medical X-ray Images Classification Based on Shape Features and Bayesian Rule." In Computational Intelligence and Communication Networks (CICN), 2012 Fourth International Conference on, pp. 369-373. IEEE, 2012.

[18] Zare, Mohammad Reza, Woo Chaw Seng, and Ahmed Mueen. "AUTOMATIC CLASSIFICATION OF MEDICAL X-RAY IMAGES." Malaysian Journal of Computer Science 26 (2013): 1.

[19] Ghofrani, Fatemeh, Mohammad Sadegh Helfroush, Mahmoud Rashidpour, and Kamran Kazemi. "FuzzyBased Medical X-ray Image Classification." Journal of medical signals and sensors 2, no. 2 (2012): 73. 